\title{
Integración de la Responsabilidad Social (SR) en los planes de estudio universitarios: un estudio de caso
}

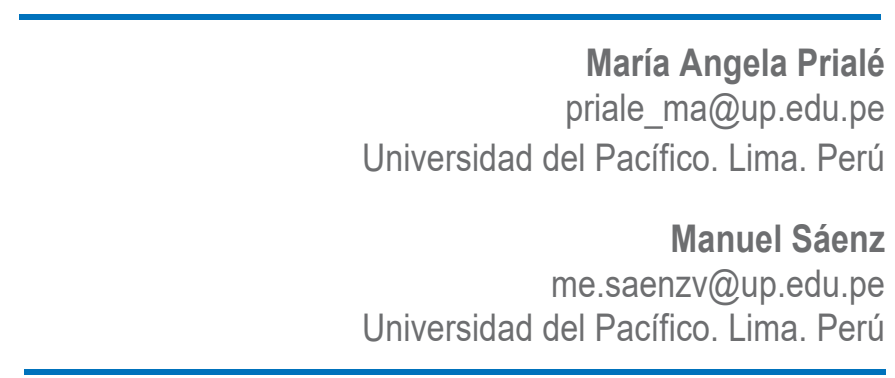

\section{Resumen}

El documento que se presenta a continuación combina la revisión de literatura existente en el campo de la enseñanza de la ética y la responsabilidad social con un estudio de caso a través del cual se analiza la integración de la responsabilidad social, de forma transversal, en las mallas curriculares de las carreras de pregrado que ofrece una universidad peruana especializada en economía y negocios. Se creó un método de análisis que explota el concepto de responsabilidad social definido en la norma ISO 26000, para generar ítems que permiten evaluar el abordaje de la responsabilidad social en los contenidos de 215 cursos obligatorios ofrecidos por los distintos departamentos académicos de la universidad. La revisión de los cursos fue realizada usando el juicio de cinco expertos. El primer aporte de esta investigación consiste en el diseño de un método transferible y replicable que permite mapear si una competencia genérica, como es la responsabilidad social, se desarrolla de forma gradual a lo largo de todo el proceso formativo. Por otro lado, del estudio de caso se desprende que el $21 \%$ de los cursos ofrecidos abordan alguna de las materias de responsabilidad social

\section{Palabras clave:}

Responsabilidad social empresarial, ética, enseñanza de responsabilidad social, enseñanza de ética. 


\section{Introducción}

La necesidad de establecer marcos y practicas éticas en los negocios es cada vez más urgente (Cornelius et al, 2007). Una creciente lista de escándalos corporativos ha generado mayor preocupación por el comportamiento destructor de valor al interior de los equipos gerenciales de las empresas y, por la responsabilidad que, en ese tema, pueden estar teniendo las escuelas de negocio a cargo de la formación de los gestores de las empresas (Valleays, 2003; Mitroff, 2004; Balotsky y Steingard, 2006; Brooks, 2009; Allens, 2009). En América Latina se ha asentado una tradición de inestabilidad, tanto política como social; reflejada en los altos niveles de corrupción y debilidad institucional y en la falta de respeto hacia las leyes. Las malas prácticas, resultado de una escasa conciencia ética de los empresarios, están guiando a esta región hacia un progreso meramente "científico" y "económico" que no toma en cuenta el desarrollo humano (Valleys, 2003).

El panorama descrito lleva a muchos a concluir que en América Latina la educación ética no está funcionando (Jaen, Rificco y Ossa, 2014). De acuerdo con Pfeffer y Fong (2004), las escuelas de negocios son responsables de proveer al mercado profesionales entrenados en principios y fundamentos éticos y de responsabilidad social. Incluso la Association to Advance Collegiate Schools of Business (AACSB), requiere que todas sus instituciones asociadas incluyan cursos de ética y responsabilidad social en sus planes de estudios. Además, recomiendan realizar un seguimiento de los resultados de aprendizaje logrados por sus estudiantes a partir de la inclusión de dichos contenidos (Waples et al., 2008).

Los objetivos que cada universidad persigue con la impartición de dichas materias difieren de una universidad a otra (Powers y Vogel, 1980, Callahan, 1980) pero, pueden agruparse en tres tipos 1) de concientización o sensibilización a los estudiantes acerca de la problemática de la ética y la responsabilidad social; 2) de fortalecimiento de habilidades para reconocer y resolver dilemas éticos (Weber, 1990) $\mathrm{y} ; 3)$ de integración de los dos objetivos anteriores en uno es decir, los cursos de ética y responsabilidad social buscarian que los estudiantes sean más sensibles a la problemática ética $\mathrm{y}$, al mismo tiempo, que decidan usando el razonamiento ético (Marnburg, 2003).

Para el logro de los objetivos mencionados las universidades que ofrecen carreras vinculadas a los negocios suelen optar entre dos modelos (McDonald, 2004). El primero es el del curso único, generalmente obligatorio, que se dicta durante un semestre académico. El segundo consiste en una aproximación más detallada en la que la ética y la responsabilidad social están completamente integradas o "transversalizadas" en toda la malla curricular.

Este segundo enfoque es el que ha recibido mayor aceptación en los últimos años (McDonald, 2004). Sin embargo, no existe un método claro que nos permita medir si efectivamente ese ideal de integración de la responsabilidad social en la currícula se está llevando a la práctica. En todo caso, ese debiera ser el punto de partida para, en una etapa posterior, evaluar si ese es el modelo más eficiente para el logro de los objetivos detrás de la enseñanza de la ética y de la responsabilidad social, que cada institución educativa haya definido. 
El estudio que se presenta a continuación pretende desarrollar un método transferible y replicable que llene el vacío mencionado. El método será probado a través del estudio de caso de una universidad peruana especializada en economía y negocios.

\section{Marco conceptual}

-Modelos para la enseñanza de la ética y la responsabilidad social: ¿Curso único o integración de la RS en toda la malla curricular?

Para atender las expectativas de la sociedad, la mayor parte de escuelas líderes, ha introducido cursos de ética y responsabilidad social en sus mallas curriculares (Fernandez y Bajo, 2010; Cornelius et al, 2007; Jones Christensen et al., 2007). Alrededor de la mitad de las universidades han optado por indicar la obligatoriedad en el cursado de esas asignaturas (Singh, 1989) y; escogido el uso de métodos innovadores de enseñanza como son el método de casos, el aprendizaje experiencial, la participación en programas sociales y el voluntariado en ONG's en busca de mayor efectividad en el proceso enseñanza-aprendizaje (Farber, V. et. al., 2015; Christensen et al. 2007, Jaen et al., 2014).

Aunque se sostiene que después de llevar un curso de ética o responsabilidad social se produce un incremento del razonamiento ético y del pensamiento crítico de los estudiantes (Abdolmohammadi y Reeves, 2000; Balotsky y Steingard, 2006; Allens, 2009), los modelos de cursos únicos han sido criticados principalmente por promover, según sus detractores, la separación de las consideraciones éticas del mundo real (Kolhberg, 1988) y, por lo tanto fallar en la generación de comportamientos éticos y responsables.

En cuanto a los modelos de integración de la RS en toda la malla curricular, se señala que su principal beneficio consiste en exponer a los alumnos a los principales problemas éticos del mundo real, tomando en consideración diferentes perspectivas. De esa manera se abordaría la resolución de distintos dilemas bajo distintas aproximaciones, trabajadas por distintos profesores (Mc Donald, 2004). Una educación integral sobre ética en los negocios debe producir competencias cognitivas, conocimientos intelectuales con los cuales los alumnos puedan formular juicios adecuados sobre la dimensión ética y la actividad económica; competencias conductuales, que se basan en la conciencia moral y; por último, competencias gerenciales, que permite extender las dos competencias anteriores al campo de los negocios (Rossouw, 2001). La tendencia hacia el modelo de integración probablemente obedezca al reconocimiento de que la enseñanza de responsabilidad social debe ser proactiva y no solo reactiva, es decir no se debe limitar a las normas, requerimientos y responsabilidades sociales y legales, sino que se debe enmarcar en un marco de pensamiento crítico mucho más amplio (Cornelius, Wallace y Tassabehji, 2007).

En la actual práctica de las escuelas de negocios, el modelo integrado aparece como la norma y como objetivo (Dunfee y Robertson, 1988; Gandz y Hayes, 1988). La mayoría de escuelas y universidades líderes dicen integrar ética y responsabilidad social en el corazón de sus currículos, otras combinan un curso sobre la materia con la 
integración de la misma en los cursos nucleares de sus planes de estudio. Sólo algunas pocas aún mantienen un modelo formativo de curso único (Woo, 2003). Sin embargo, poco se ha analizado si esa deseada integración es real.

-Construyendo la integración o "transversalizando" la enseñanza de la ética y la responsabilidad social

Asumiendo que el modelo de integración es el más completo, su aplicación también resulta retadora pues para poner operativo el modelo se requiere una revisión exhaustiva del currículo, la capacitación de los docentes y el monitoreo permanente de los resultados. En ese sentido, la "transversalización" de la RS supondría una integración plena de los conocimientos éticos en el análisis y elección de cursos de acción dentro de todas las áreas disciplinarias del plan de estudios (Gandz y Hayes, 1988). También requeriría que ética y responsabilidad social sean "enseñadas" en varios cursos, incluso aquellos que abordan el desarrollo de competencias técnicas.

En lo que respecta a la revisión del currículo en un primer momento, se debe tener en cuenta la necesidad de contar con un diagnóstico o línea de base sobre la situación actual de la oferta formativa. Dicho diagnóstico deberá empezar por reconocer el objetivo de enseñar ética y responsabilidad social que persigue la universidad. En ese sentido, un objetivo que suele mencionarse es integrar la competencia de gestión con la competencia moral (Powers y Vogel, 1980). Luego de definido el objetivo el mayor desafío radica en encontrar la forma adecuada para alcanzarlo.

En relación con la capacitación de los docentes se sugiere que para enseñar ética en los negocios se debe conocer la teoría ética y las particulares estructuras y contexto que son únicos para tomar decisiones éticas dentro del mundo de los negocios (Morse, 1999). Debido a que ese perfil no es frecuente entre los profesores, especialmente en aquellos centrados en el desarrollo de competencias técnicas es probablemente que resulte necesario formar a los filósofos en contenidos de gestión empresarial y a los docentes de materias de gestión en ética y responsabilidad social. Además, de esa tipo de formación, habrá que trabajar en dotarlos de las herramientas pedagógicas necesarias para inculcar valores y cambiar actitudes de los estudiantes (Josephson, 1988; Hunt and Bullis, 1991). Los educadores necesitan atender el desarrollo de métodos de enseñanza adecuados para el desarrollo de competencias genéricas como son la ética y la responsabilidad social (Datar et al., 2011; Rossouw, 2002; Balotsky and Steingard, 2006; Alsop, 2003; Hartman and Hartman, 2005).

Finalmente, en cuanto a la medición de resultados, Loeb (1991) propone un modelo de evaluación de "outcomes" en la enseñanza de ética en la carrera de contabilidad, el cual puede ser extendido a cualquier carrera de negocios. Este modelo es una evaluación integral puesto que considera a alumnos, profesores y el análisis de los cursos. La evaluación a los alumnos emplea técnicas clásicas como son exámenes, evaluación de casos, participación en clase y trabajos grupales. La evaluación a los docentes sería mediante encuestas a los alumnos. Por último, los programas de los cursos deberán ser analizados para ver si sus objetivos están alineados con los objetivos del plan de estudios.

En relación con los puntos anteriores se ha identificado que para el levantamiento de la línea de base que sirva como punto de partida para transformar el modelo de curso 
único de responsabilidad social hacia el integral, no existe una herramienta transferible y confiable que permita medir si la responsabilidad social se está integrando en la malla curricular. Si bien algunos autores ofrecen recomendaciones sobre el proceso a seguir (McDonald, 2004) éstas son genéricas y no presentan recursos para la sistematización de la información de diagnóstico. Este paso es crucial para la correcta ejecución de todo el proceso de integración ya sea dentro de todos los cursos o en cursos core específicos.

En la siguiente sección, poniendo como ejemplo el caso de una universidad peruana especializada en economía y negocios se propondrá una herramienta metodológica para facilitar el diagnóstico y llenar dicho vacío.

\section{Caso: propuesta de un método para el levantamiento de línea de base de la integración de la RS en la malla curricular}

\section{-Antecedentes}

La Universidad analizada está localizada en Lima, Perú, se trata de una institución especializada en Economía y Negocios tanto a nivel de Pregrado como de Postgrado. Acreditada por la AACSB, desde sus orígenes, en 1962, ha hecho explícito su compromiso con el desarrollo del país. La misión de la Universidad se resume en la formación de líderes responsables promotores del desarrollo sostenible de su país y el mundo.

Con respecto a la organización de la oferta formativa, son los departamentos académicos los proveedores de las asignaturas a todas las carreras. Existen siete Departamentos Académicos: Administración, Contabilidad, Economía, Derecho, Ingeniería, Ciencias Sociales y Humanidades encargados de la provisión de cursos de acuerdo con las necesidades de cada carrera.

El modelo de enseñanza de ética y responsabilidad social elegido por la Universidad, fue hasta 2011 el de cursos aislados. El plan de estudios aprobado en 2005 incluyó como asignatura obligatoria para las carreras de administración, contabilidad, marketing y negocios internacionales, el curso Gestión estratégica de la responsabilidad social. Además, desde la fundación de la universidad, el curso de Moral Profesional era obligatorio para todas las carreras.

En 2011 se realizó un estudio para conocer la percepción que los estudiantes de la carrera de administración tenían de sus aprendizajes con dichas asignaturas y, el grado en el que ellos consideraban que la responsabilidad social y la ética eran cultivadas a lo largo de toda su permanencia en la universidad. Los resultados arrojaron que la mayoría de estudiantes alcanzaba estadios intermedios en el logro del resultado de aprendizaje esperado es decir, eran más sensibles al papel que la empresa debe cumplir en la sociedad y manejaban de forma adecuada conceptos pero, sólo el $25 \%$ manifestaba haber desarrollado un razonamiento de responsabilidad social en la toma de decisiones profesionales. Además, los estudiantes señalaron que existía inconsistencia entre el mensaje recibido en los cursos técnicos de su carrera que sobrevaloraban la maximización de beneficios económicos en el corto plazo y, el 
que se transmitía en el curso de Responsabilidad Social, la preocupación por la triple cuenta de resultados: económico, social y ambiental.

Las conclusiones mencionadas llevaron a la universidad a tomar dos tipos de acciones: 1) revisión de los objetivos, contenido y estrategia didáctica del curso en cuestión y, 2) formación en la materia, a los docentes de los cursos técnicos que se consideraron más relacionados con algún tópico de la responsabilidad social. La meta, especialmente con la segunda acción, fue sentar las bases para un proceso de migración a modelo integrado en la enseñanza de la responsabilidad social.

Además, de las acciones mencionadas, para tener una imagen realista del estado de la integración de la responsabilidad social en las mallas curriculares se desarrolló y aplicó un método basado en la revisión de los sílabos. El sílabo es un contrato entre el profesor y el alumno, en dicho "contrato" el primero se compromete con el segundo a cumplir con el desarrollo de los temas consignados en el documento. Por ello, se presupone que todos los temas contenidos en los sílabos son tratados en clase de manera explícita. En consecuencia se considera que a través del análisis de sílabos se pueden obtener datos objetivos acerca de la presencia de la responsabilidad social en asignaturas distintas a las diseñadas específicamente para formar en esa materia. Por tanto, a través de la revisión de los sílabos se buscó dimensionar el grado en el que la responsabilidad social está integrada en la malla curricular.

\section{-Método}

Se optó por una investigación exploratoria pues su objetivo es examinar un tema o problema de investigación poco estudiado, del cual se tiene muchas dudas o no se ha abordado antes para indagar temas desde nuevas perspectivas (Hernández et al 2006). Ello pasa por crear un instrumento no encontrado en la literatura que permita determinar el grado en el que la responsabilidad social se integra en la malla curricular.

Específicamente se optó por el método de estudio de caso único, adecuado para la universidad elegida debido a que esta representa un caso revelatorio (actualmente no existen bases de datos que hayan recolectado los sílabos de todos los cursos de una universidad). Se considera también como un caso representativo debido a que en la misión de la universidad se puede encontrar un componente de responsabilidad social (Yin, 2009).

Se analizó un total de 215 sílabos, correspondientes a 188 cursos entre obligatorios y electivos, ofrecidos por los 7 departamentos académicos. Los sílabos corresponden a los cursos que fueron dictados el semestre 2013-II (Ciclo de Agosto a diciembre del 2013).

Los cursos sílabos están distribuidos como se muestra en la Tabla 1. Cabe resaltar que un mismo curso dictado por varios profesores distintos puede tener diferentes temas abordados, por esta razón, en 18 asignaturas se analizaron sílabos de distintos profesores que dictan el mismo curso.

Para construir el instrumento de recogida de datos, se partió del concepto de responsabilidad social desarrollado en la norma ISO 26000 que la define como "la 
responsabilidad de una organización ante los impactos que sus decisiones y actividades ocasionan en la sociedad y el medio ambiente, mediante un comportamiento ético y transparente que: tome en consideración los intereses de sus partes interesadas; cumpla con la legislación aplicable y sea coherente con la normativa internacional de comportamiento; y esté integrada en toda la organización y se lleve a la práctica en sus relaciones" (ISO 26000, Cap. 2,Apdo. 2.18).

Tabla 1. Distribución de los sílabos analizados por departamento académico
\begin{tabular}{lcc} 
Departamentos & $\begin{array}{c}\text { Número total de sílabos } \\
\text { del departamento } \\
\text { académico analizados }\end{array}$ & $\%$ \\
\hline Administración & 49 & $23 \%$ \\
Economía & 44 & $20 \%$ \\
Derecho & 39 & $18 \%$ \\
Contabilidad & 35 & $16 \%$ \\
Ingeniería & 28 & $13 \%$ \\
Humanidades & 15 & $7 \%$ \\
Ciencias Sociales & 5 & $2 \%$ \\
\hline Total & 215 & $100 \%$ \\
\hline
\end{tabular}

Se considera la definición de la RS de la norma ISO 26000 como un constructo válido para evaluar la integración de la responsabilidad social en las mallas curriculares ya que dicha definición nace de un consenso global de expertos construido en diez años de trabajo y que contó con la participación de distintos grupos de interés de todas las regiones del mundo. Dicho consenso se dio en tormo a: 1) qué principios, materias fundamentales y asuntos de responsabilidad social deberían ser tenidos en cuenta por cualquier organización; y, 2) qué debería hacer la organización para poner en práctica la RS. En este sentido, la ISO 26000 logra sintetizar una gran diversidad de criterios en una sola norma internacional, coherente y al alcance de todos.

La norma también señala siete materias fundamentales relacionadas con la RS. Para cada una de estas materias se detallan entre una y cinco dimensione asociadas. Estas materias son, de algún modo, los ámbitos en los que la organización debe centrar su atención a la hora de tratar de actuar responsablemente.

Luego de revisar en detalle la descripción de cada una de las materias y las dimensiones que incluye se pasó a la construcción de palabras y términos clave que se utilizarían para validar si en los sílabos están presentes dichas dimensiones. Un extracto de la plantilla de recogida de datos que incluye materias y dimensiones de la responsabilidad social se presenta en la tabla 2.

Como se mencionó, para el análisis y procesamiento del contenido de los sílabos se decidió usar términos clave, dichos términos clave se utilizaron para identificar dimensiones de responsabilidad social y se encuentran recogidos en una guía de sistematización presentada en el Apéndice 1.

Ya que a pesar del uso de la guía la identificación de contenido de responsabilidad social en las mallas curriculares puede estar sujeto a subjetividad (multiplicidad de conceptos inmersos la definición misma de responsabilidad social) el procesamiento 
de los contenidos se llevó a cabo y validó de acuerdo con la propuesta metodológica de juicio de expertos elaborada por Cooke y Goossens (1999).

Tabla 2. Plantilla de sistematización de los sílabos

\begin{tabular}{|c|c|c|c|c|c|}
\hline \multicolumn{2}{|r|}{ Responsabilidad social } & \multicolumn{2}{|c|}{ Dpto. Administración } & \multicolumn{2}{|c|}{ Dpto. Contabilidad } \\
\hline Dimensión & Variable & Adm 01 & Adm 02 & Cont 01 & Cont 02 \\
\hline Gobernanza de la organización & Promoción de la responsabilidad social & & & & \\
\hline \multirow[t]{8}{*}{ Derechos humanos } & Debida diligencia & & & & \\
\hline & Situaciones de riesgo para los derechos humanos & & & & \\
\hline & Evitar la complicidad & & & & \\
\hline & Resolución de reclamaciones & & & & \\
\hline & Discriminación y grupos vulnerables & & & & \\
\hline & Derechos civiles y politicos & & & & \\
\hline & Derechos económicos, sociales y culturales & & & & \\
\hline & Principios y derechos fundamentales en el trabajo & & & & \\
\hline \multirow[t]{5}{*}{ Prácticas laborales } & Trabajo y relaciones laborales & & & & \\
\hline & Condiciones de trabajo y protección social & & & & \\
\hline & Diálogo social & & & & \\
\hline & Salud y seguridad ocupacional & & & & \\
\hline & Desarrollo humano y formación en el lugar de trabajo & & & & \\
\hline \multirow[t]{4}{*}{ El medio ambiente } & Prevención de la contaminanción & & & & \\
\hline & Uso sostenible de los recursos & & & & \\
\hline & Mitigación y adaptación al cambio climático & & & & \\
\hline & $\begin{array}{l}\text { Protección del medio ambiente, la biodiversidad y la } \\
\text { restauración de hábitats naturales }\end{array}$ & & & & \\
\hline \multirow[t]{5}{*}{ Prácticas justas de operación } & Anti-corrupción & & & & \\
\hline & Participación política responsable & & & & \\
\hline & Competencia justa & & & & \\
\hline & Promover la responsabilidad social en la cadena de valor & & & & \\
\hline & Respeto a los derechos de la propiedad & & & & \\
\hline \multirow[t]{7}{*}{ Asuntos de consumidores } & $\begin{array}{l}\text { Prácticas justas de marketing, información objetiva en } \\
\text { imparcial y prácticas justas de contratación }\end{array}$ & & & & \\
\hline & \begin{tabular}{|l} 
Protección de la salud y seguridad de los consumidores \\
\end{tabular} & & & & \\
\hline & Consumo sostenible & & & & \\
\hline & Servicios de Atención & & & & \\
\hline & Protección de la privacidad & & & & \\
\hline & Acceso a servicios esenciales & & & & \\
\hline & Educación y toma de conciencia & & & & \\
\hline \multirow[t]{7}{*}{$\begin{array}{l}\text { Particiipación activa y desarrollo } \\
\text { de la comunidad }\end{array}$} & Participación activa de la comunidad & & & & \\
\hline & Educación y cultura & & & & \\
\hline & Creación de empleo y desarrollo de habilidades & & & & \\
\hline & Desarrollo y acceso a la tecnología & & & & \\
\hline & Generación de riqueza e ingresos & & & & \\
\hline & Salud & & & & \\
\hline & Inversión social & & & & \\
\hline
\end{tabular}

Los expertos leyeron la totalidad del sílabo, incluida la bibliografía y marcar con un 1 si la dimensión se abordaba o con un 0 si no se abordaba en el sílabo. El método de juicio de expertos siguió los siguientes pasos:

- Definición del caso: Sílabos de todos los cursos de la universidad para el ciclo de estudios 2013-II, con el fin de identificar elementos relacionados a la responsabilidad social.

- Identificación de variables: Se seleccionaron palabras calve relacionadas con las 7 materias fundamentales de la ISO 26000 (ISO, 2010), las cuales son: gobernanza de la organización, derechos humanos, prácticas laborales, medio ambiente, prácticas de justa operación, asuntos de consumidores, y participación activa y desarrollo de la comunidad. Cada una de estas materias se subdivide en varias dimensiones. 
- Identificación de expertos: Se evaluó a un grupo de expertos, los cuales participan activamente en investigaciones en temas de responsabilidad social y poseen un buen manejo de la ISO 26000 (ISO, 2010). Al final, se seleccionó a un grupo de 5 expertos.

- Sesión de entrenamiento de expertos: Se realizó una guía de sistematización, la cual ahondó en el contenido de las 7 materias fundamentales ya mencionadas y contenía una lista de palabras clave, las cuales fueron las que se buscaron, o sus sinónimos, en los sílabos. Se revisó junto con los expertos dicha guía y se realizó junto con ellos un ejercicio de preparación.

- Procesamiento: Se le entregó a cada uno de los expertos una plantilla que cruzaba los cursos por departamento académico con las 7 materias fundamentales de la responsabilidad social y las dimensiones contenidas en cada uno. El trabajo de los expertos fue identificar si el contenido de alguna de las dimensiones de las materias de la responsabilidad social se encuentra de manera explícita en el sílabo.

Se consideró como abordada alguna de las dimensiones de las materias de responsabilidad social si un mínimo de cuatro de los cinco expertos $(80 \%)$ la identificaron dentro del sílabo correspondiente, cifra consistente de acuerdo a Zaichkowsky (1994) y Hernani y Hamann (2012).

\section{Resultados}

Como se puede observar en la Tabla 3 . El $21 \%$ de todos los sílabos de los cursos dictados en la Universidad en el ciclo de estudios 2013-II abordaron por lo menos alguna dimensión/materia de la responsabilidad social.

Tabla 3. Distribución por departamento académico de los cursos que tratan por lo menos una materia de la responsabilidad social

\begin{tabular}{lccc}
\hline Departamentos & $\begin{array}{c}\text { Número total de sílabos del } \\
\text { departamento académico } \\
\text { analizados }\end{array}$ & $\begin{array}{c}\text { Número de sílabos del } \\
\text { departamento académico } \\
\text { que abordan RS }\end{array}$ & $\begin{array}{c}\% \text { de sílabos del } \\
\text { departamento } \\
\text { académico que } \\
\text { abordan RS }\end{array}$ \\
\hline Administración & 49 & 17 & $35 \%$ \\
Economía & 44 & 3 & $7 \%$ \\
Derecho & 39 & 17 & $44 \%$ \\
Contabilidad & 35 & 1 & $3 \%$ \\
Ingeniería & 28 & 2 & $7 \%$ \\
Humanidades & 15 & 2 & $13 \%$ \\
Ciencias & 5 & 3 & $60 \%$ \\
Sociales & 215 & 45 & $21 \%$ \\
\hline Total & & & \\
\hline
\end{tabular}

Con respecto a la distribución por departamento académico, Administración y Derecho son los que incluyen mayor cantidad de cursos que abordan al menos una materia de 
la responsabilidad social, esto en cantidades absolutas. Por otro lado, en nivel porcentual, el departamento de ciencias sociales ocupa el primer puesto con $60 \%$ de sus cursos (tres de cinco) que abordan por lo menos alguna materia. Cabe resaltar la poca cantidad de cursos pertenecientes a los departamentos de Economía, Contabilidad e Ingeniería que impartes cursos con contenido de responsabilidad social, tres y uno respectivamente.

El departamento académico de Administración ofrece una gran cantidad de cursos que abordan las materias de Gobernanza de la organización (nueve cursos) y prácticas laborales (ocho cursos), en especial temas de desarrollo humano y relaciones laborales. Esto se puede ver en la lista de cursos del Anexo 3 que muestra ocho cursos sobre manejo de recursos humanos y desarrollo del talento como: Relaciones laborales, Gestión de personas, Retención y compensación del talento, Gestión de personas y Gestión del capital humano. El tema de Gobernanza en la organización estaría presente de manera transversal en varios cursos de este departamento. Los cursos ofrecidos por este departamento son parte de las mallas de carreras como administración, marketing, negocios internacionales y contabilidad. Este departamento también ofrece cursos a las carreras de ingeniería, economía, finanzas y derecho.

En el caso de los cursos ofrecidos por el departamento académico de Derecho, se ve que 12 cursos (31\%) abordan temas de derechos humanos, más específicamente temas de derechos civiles y laborales. Estos contenidos están ligados a los cursos correspondientes al derecho civil y al derecho laboral. Este departamento también ofreció dos cursos que abordan el tema de gobernanza en la organización. En el caso de los cursos impartidos por el departamento académico de Derecho, la mayoría de estos son correspondientes exclusivamente a la carrera de derecho.

Los departamentos de Ingeniería, Contabilidad y Ciencias sociales ofrecen un curso cada uno que aborda el tema de gobernanza de la organización. Adicionalmente los departamentos de Humanidades y Ciencias sociales ofrecen dos cursos cada uno que aborda temas de derechos humanos. Es importante notar que los cursos de estos dos departamentos son impartidos a todas las carreras, por lo tanto el contenido de responsabilidad social de los mismos es transversal a las mallas de las distintas carreras. El departamento académico de Economía solo imparte tres cursos que abordan contenidos de responsabilidad social: dos de estos abordan materias del medio ambiente y uno aborda temas de desarrollo de las comunidades.

Los resultados evidencian que los departamentos que ofrecen una mayor cantidad de cursos que abordan temas de responsabilidad social son los de Administración y Derecho. En el caso de los cursos ofrecidos por el departamento de derecho, al estar estos incluidos en las mallas de varias carreras, se podría concluir que al sumarlo con los cursos ofrecidos por los demás departamentos, en la mayoría de carreras se podrá encontrar una presencia mediana de responsabilidad social a lo largo de sus años de estudio. 


\section{Conclusiones y futuras líneas de investigación}

En el caso específico presentado, el método propuesto ha probado ser útil para diagnosticar objetivamente la integración explícita de la responsabilidad social en los distintos cursos de las mallas curriculares. Este puede ser un inicio del proceso que permita monitorear el nivel de avance en el objetivo de "transversalizar" o integrar la enseñanza de la responsabilidad social. Incluso, métodos similares podrían proponerse para evaluar la integración de otras competencias genéricas a lo largo de todos los planes de estudio. No obstante, para validar el método sería deseable probar el desempeño de la herramienta en otras universidades.

Para emitir un juicio valorativo acerca del nivel de transversalidad de la responsabilidad social en los programas formativos de las universidades es necesario incorporara variables más cualitativas que no han sido tomadas en cuenta en ese estudio. Así, para que la integración sea real será necesario trabajar con los docentes. Más allá de los contenidos señalados en los sílabos, el papel del profesor es fundamental. Si el docente no maneja adecuadamente métodos de enseñanza adaptados para el abordaje de la RS o su dominio técnico de la materia es intuitivo se corre el riesgo de que a pesar del compromiso sellado en el sílabo de trabajar en la materia, la adquisición de la competencia por parte del estudiante no se produzca. De otro lado, también es cierto que algunos docentes incluyen en el desarrollo de sus cursos la reflexión ética y de responsabilidad social sin hacerla explícita en su sílabo. Este tipo de consideraciones y de análisis deben acompañar la reflexión acerca del plan de transversalización.

Otra consideración es que si bien en este trabajo hemos optado por un análisis en función a departamentos académicos, los datos recogidos podrían presentarse por carrera. Esto ayudaría a establecer el perfil de los cursos en los que se están trabajando materias de responsabilidad social así podrían identificarse nuevos cursos en función a si el objetivo de integración es en toda la malla o solo en algunos cursos core que pueden considerarse más alineados a determinadas materias.

El análisis de la integración de la responsabilidad social a la luz de la norma ISO 26000 es completo y útil pues incluye variables relacionadas con las expectativas que los distintos grupos de interés y la sociedad en general tienen acerca de las empresas. Ello tiene una ventaja, ofrece una orientación clara acerca de los temas que se espera que los profesionales formados en las universidades consideren al tomar decisiones de negocios.

\section{Referencias bibliográficas}

Allens Jones, D. (2009). A Novel Approach to Business Ethics Trainning: Improving Moral Reasoning in Just a Few Weeks. Journal Of Business Ethics 88, 367-379.

Alsop, R. (Setiembre de 2003). Right and Wrong: Can Business Schools Teach Students to be Virtous? Wall Street Journal. 
Balotsky, E., \& Steingard, D. (2006). How Teaching Business Ethics Makes a Difference: Findings from an Ethical Learning Model. Journal of Business Ethics Education 3, 5-34.

Brooks, A. (2009). Business schools mull over blame in financial crisis. Recuperado el 19 de Diciembre de 2014, de NPR: http://www.npr.org/templates/story/story.php?storyld=103719186

Callahan, D. (1980). Goals in Teaching Ethics. En D. Callahan, \& S. Bok, Ethics in Higher Education (págs. 547-551). New York: Plenum Press.

Christensen, L. J., Peirce, E., Hartman, L. P., Hoffman, W. M., \& Carrier, J. (2007). Ethics, CSR and Sustainability Education in the Financial Times Top 50 Global Business Schools:Baseline Data and Future Research Direction. Journal of Business Ethics 73, 347-368.

Cooke, R., \& Goossens, L. (1999). Procedures guide for structured expert judgement. Luxemburgo/Bruselas: European Commission Community Research.

Cornelius, N., Wallace, J., \& Tassabehji, R. (2007). An Analysis of Corporate Social Responsibility, Corporate Indetity and Ethics Teaching in Business Schools. Journal of Business Ethics, 76, 117-135.

Datar, S., Garvin, D., \& Cullen, P. (2011). Rethinking the MBA: business education at a crossroad. Journal of Management Development, 30(5), 451-462.

Dunfee, T., \& Robertson, D. (1988). Integrating Ethics into the Business School Curriculum. Journal of Business Ethics, 7, 847-859.

Farber, V., Prialé, M., \& Fuchs, R. (2013). An Entrepreneurial learning exercise as a pedagogical tool for teaching CSR. Industry \& Higher Education, 29(5), 1-16.

Fernandez Fernandez, J. L., \& Bajo Sanjuán, A. (2010). The Presence of Business Ethics and CSR in Higher Education Curricula for Executives: The Case of Spain. Journal of Business Ethics Education 7, 25-38.

Gandz, J., \& Hayes, N. (1988). Teaching Business Ethics. Journal of Business Ethics, 7, 657-669.

Hartman, L., \& Hartman, E. (2005). How to Teach Ethics: Assumptions and Arguments. Journal of Business Ethics Education, 1, 162-212.

Hernández, R; Fernandez C; Baptista, P. Metodología de la Investigación. 4. ed. México: McGraw-Hill, 2006.

Hernani, N.M \& Hamann , A.(2013).Percepción sobre el desarrollo sostenible de las Mype en el Perú. RAE, Sao Paulo, 53 ( 3), 290-302

Hunt, E., \& Bullis, R. (1991). Applying the Principles of Gestalt Theory to Teaching Ethics. Journal of Business Ethics, 10(5), 341-347.

ISO. (2010). Norma Internacional ISO 26000. Ginebra. 
Jaén, M. H., Reficco, E., \& Ossa, M. (2014). Can civility be taught to Latin-American MBAs? Implications of case-method use for the development of civic behaviour in managerial education. Management Decision 52(9), 1833-1856.

Josephson, M. (1988). Teaching ethical decision making and principled reasoning. Ethics:Easier Said Than Done, 27-33.

Loeb, S. E. (1991). The Evaluation of "Outcomes" of Accounting Ethics Education. Journal of Business Ethics 10, 77-84.

Marnburg, T. R. (2003). Educational Impacts on Academic Practitioner's Moral Reasoning and Behaviour: Effects of Short Courses in Ethics or Philosophy. Business Ethics: A european Review 12 (4).

McDonald, G. M. (2004). A Case Example: Integratinf Ethics into the Academic Business Curriculum. Journal Of Business Ethics 54, 371-384.

Mitroff, I. (2004). An open letter to the deans and the faculties of American Business Schools. Journal of Business Ethics, 81(4), 185-189.

Morse, J. (1999). Who is the Ethics Expert? The original footnote to Plato. Business Ethics, 9(4), 693-697.

Pfeffer, J., \& Fong, C. (2004). The Business Schools "Business": Some Lessons from the US experience. Journal of Management Studies, 54(2), 185-189.

Powers, C., \& Vogel, D. (1980). Ethics in the Education of Business Managers. (Henderson, Ed.) Sloan Management Review, 39-49.

Rossouw, G. (2002). Three Approaches to Teaching Business Ethics. Teaching Business Ethics 6(4), 411-433.

Singh, J. (1989). The Teaching of Ethics in Canadian Schools of Management and Administrative Studies. Journal of Business Ethics, 8(1), 51-56.

Valleys, F. (2003). Orientaciones para la enseñanza de la ética, el capital social y el desarrollo en las universidades latinoamericanas. Revista Venezolana de Gerencia 21, 38-73.

Waples, E., Antes, A., Murphy, S., Connelly, S., \& Mumford, M. (2008). A Meta-analytic Investigation of Business Ethics Instruction. Journal of Business Ethics, 87(1), 133-151.

Woo, C. (2003). Personally Responsible: The Basis of an Ethics Education Revolves Around Teaching Business Students a Sense of Individual Responsibility. BizEd, 2(4), 22-27.

Weber, J. (1990). Mesuring the Impact of Teaching Ethics to Future Managers: A review, Assesment and Recommendations'. Journal of Business Ethics,9(3), 183-190.

Yin, R. K. (2009). Case Study Research: design and methods. New Delhi: SAGE.

Zaichkowsky, J. (1994). The Personal Involvement Inventory: Reduction, Revision, and Application to Advertising. Journal of Advertising, 23(4), 59-70. 


\section{Apéndice 1.}

-Guía de sistematización de los sílabos

La sistematización de los sílabos se llevará a cabo tomando como base las 7 materias fundamentales de la responsabilidad social definidas en la ISO 26000: Gobernanza de la organización, derechos humanos, prácticas laborales, medio ambiente, prácticas de justa operación, asuntos de consumidores, y participación activa y desarrollo de la comunidad. Estas materias se consideran dimensiones del término responsabilidad social.

Las variables en las que se desagregan cada una de las dimensiones de la RSE corresponden a los asuntos que, de acuerdo con la ISO 26000, deben gestionar las organizaciones socialmente responsables.

El objetivo del investigador es obtener, luego de la lectura detallada de los acápites de los sílabos (objetivos, contenidos, bibliografía, resultado de aprendizaje, actividades a desarrollar por el estudiante a la largo del curso) un mapa que permita localizar los cursos que abordan variables constituyentes de la RSE.

Para reducir la subjetividad, el procedimiento que deberá seguir el investigador es marcar en la plantilla de recolección de datos, un 1 si el término exacto de la variable o un sinónimo del mismo (por ejemplo gobernanza=buen gobierno corporativo) se encuentra explicitado en el sílabo. Si ello no ocurriera, deberá marcar un 0 en la plantilla de recolección de datos.

Si bien es cierto la ISO 26000 es una guía de responsabilidad social con aplicación directa en la gestión, consideramos que su lógica pueda adaptarse y ser apropiada para el análisis de cursos. Por ello, se ha optado por buscar la presencia de términos clave (cada una de las variables) en los sílabos. Aunque debe advertirse que pudiera ser que la materia se trabajara en el curso bajo una "denominación distinta a la de la variable" se ha optado por buscar los términos clave para reducir al máximo la subjetividad y los sesgos de los evaluadores.

A continuación, a manera de guía para el evaluador se presentan las dimensiones/materias:

1. Gobernanza: la gobernanza es el sistema de toma e implementa decisiones para lograr sus objetivos. Incluye mecanismos formales (estructuras y procesos definidos) e informales (derivados de la cultura organizacional y los valores). Una organización que se plantea ser socialmente responsable debería contar con un sistema de gobernanza que le permita supervisar y poner en prácticas los principios de RS.

\section{Términos clave}

a. Promoción de la responsabilidad social (procesos y estructuras que promueven transparencia, comportamiento ético, rendición de cuentas, respeto a los intereses delos grupos de interés, respeto de la legalidad, 
respeto de derechos humanos, respeto normativa internacional de comportamiento)

2. Derechos Humanos: se refiere a los derechos básicos del ser humano y se divide en derechos civiles y derechos económicos, sociales y culturales.

Términos clave:

a. Debida diligencia (proceso exhaustivo y proactivo para identificar, prevenir y abordar impactos reales o potenciales sobre los derechos humanos)

b. Situaciones de riesgo para los derechos humanos (identificación de situaciones como conflictos, inestabilidad política, proximidad a comunidades indígenas, cultura de corrupción)

c. Evitar la complicidad (ayudar o instigar un acto ilícito u omisión de los derechos humanos)

d. Resolución de reclamaciones (mecanismos para resolver reclamos en torno a los derechos humanos)

e. Discriminación a grupos vulnerables (por razones de raza, religión, género, etnia, nacionalidad, edad, origen, etc.)

f. Derechos civiles y políticos (a la vida, a una vida digna, a librarse de la tortura, seguridad de las personas, propiedad, de libertad de opinión, de reunión pacífica, de pensamiento, etc.)

g. Derechos económicos, sociales y culturales (trabajo, alimentación, salud, educación e igualdad social, trabajo infantil, trabajo forzado)

h. Principios y derechos fundamentales en el trabajo (libertad de asociación, eliminación de trabajo forzoso, abolición del trabajo infantil, eliminación de discriminación en acceso a empleo)

3. Prácticas laborales: comprenden todas las prácticas y políticas de trabajo dentro de una organización. Estas prácticas incluyen reclutamiento y promoción, procedimientos disciplinarios y resolución de reclamaciones, salud y seguridad en el trabajo, jornada laboral y remuneraciones, reconocimiento y dialogo con organización de trabajadores. Estas medidas se basan en la Declaración de Filadelfia de la OIT.

Términos clave:

a. Trabajo y relaciones laborales responsables (reclutamiento y promoción responsable de los trabajadores, cumplimiento de la regulación que se impone al empleador, empleo seguro, aviso razonable previo al despido, igualdad de oportunidades en el empleo, protección de privacidades de trabajadores, eliminación de prácticas de despido arbitrarias o discriminadoras).

b. Condiciones de trabajo y protección social (Salud y seguridad en el trabajo; condiciones de trabajo decentes en relación a salarios, horas de trabajo, descanso semanal, vacaciones, protección de la maternidad, conciliación de vida laboral y familiar, salarios adecuados a las 
necesidades de los trabajadores y sus familias, cumplimiento de regulación de protección social), pago de horas extra, cumplimiento de horarios laborales).

c. Diálogo social (no obstruir la formación de sindicatos u otras organizaciones de diálogo social, no obstruir la negociación colectiva, dar aviso cuando los cambios en las operaciones puedan producir impactos en el empleo, proporcionar a los trabajadores debidamente designados información y facilidades para cumplir con sus funciones de representación

d. Salud y seguridad ocupacional (control de riesgos para la salud de sus trabajadores, registro de incidentes y problemas en materia de salud y seguridad del trabajador, protección equitativa en salud

e. Desarrollo humano y formación en el lugar de trabajo (acceso al desarrollo de habilidades, formación y aprendizaje práctico, oportunidades de promoción equitativa y no discriminatoria).

4. Medio ambiente: Comprende el control de las externalidades negativas que las empresas podrían producir en el medio ambiente, sea la generación de contaminación e impacto sobre los hábitats naturales. La ISO 14000 puede proporcionar herramientas técnicas adecuadas para abordar cuestiones ambientales.

Términos clave

a. Prevención de la contaminación (identificación de fuentes de contaminación y residuos, prevención de contaminación, divulgación de información sobre impacto ambiental, plan de accidentes frente a emergencias ambientales)

b. Uso sostenible de los recursos (eficiencia en los recursos para reducir uso de energía, agua y otros recursos, promoción de compras sostenibles, promoción del consumo sostenible)

c. Mitigación y adaptación al cambio climático (reducción y control de la producción de gases de efecto invernadero)

d. Protección del medio ambiente, la biodiversidad y la restauración de hábitats naturales (valoración y protección de la biodiversidad; valoración, protección y restauración de servicios a los ecosistemas, uso sostenible de los recursos naturales, fomento de desarrollo urbano y rural ambientalmente sólido)

5. Practicas justas de operación: Se refiere a conducta ética en transacciones entre organizaciones y con otros entes como agencias gubernamentales, proveedores, clientes, competidores y asociaciones. Estas prácticas se representan en ámbitos de anticorrupción, comportamiento socialmente responsable, participación responsable en esfera pública, competencia justa, relación con otras organizaciones, respetos de derechos de propiedad. 
Términos clave:

a. Anticorrupción (sistemas de lucha contra la corrupción y el soborno, mecanismos de denuncia frente a situaciones de soborno y7o corrupción)

b. Participación política responsable (manejo de conflictos de interés, contribuciones políticas, lobby)

c. Competencia justa (fijación de precios, licitaciones fraudulentas, fijación desleal de precios, prácticas anti-monopolio, prácticas anti-dumping)

d. Promover la responsabilidad social en la cadena de valor (integración en sus políticas y prácticas de compra, distribución y contratación criterios éticos, sociales, ambientales y de igualdad de género).

e. Respeto a los derechos de propiedad (compensación justa por la propiedad que adquiere o usa, respeto de derechos de propiedad y conocimiento tradicional).

6. Asuntos de consumidores: Estas responsabilidades son con las personas que compran productos o servicios con propósitos privados. Estas incluyen proporcionar información precisa, justa, trasparente y útil de marketing y procesos de contratación; promover el consumo sostenible y el diseño de productos y servicios que proporcionen acceso a todos y satisfagan necesidades de los más vulnerables; y protección de la salud.

Términos clave:

a. Prácticas justa de marketing, información objetiva e imparcial y prácticas justas de contratación (precios transparentes, publicidad no engañosa, información completa y comprensible para la toma de decisiones, publicidad que no perpetue estereotipos)

b. Protección de la salud y seguridad de los consumidores (productos no peligrosos)

c. Consumo sostenible (productos con poco impacto negativo en el medio ambiente, información sobre impactos del producto en el medio ambiente, oferta de productos de alta calidad con una vida más larga)

d. Servicios de atención al cliente, apoyo y resolución de quejas y controversias (certificados de garantía, servicios de apoyo y asesoramiento, oferta de servicios de mantenimiento a precios razonables, atención de quejas y reclamos, compensación al consumidor).

e. Protección y privacidad de los datos de los consumidores (obtención de datos por medios legales y justos, no revelación de información del consumidor, protección de privacidad del cliente)

f. Acceso a servicios esenciales (tarifas subvencionadas, ampliación de cobertura a todos los grupos de consumidores, igualdad en el consumo, subvenciones a personas necesitadas)

g. Educación y toma de conciencia (educación a los consumidores acerca de sus derechos, riesgos, protección al medio ambiente). 
7. Participación activa y desarrollo de la comunidad: Se refiere a las contribuciones al desarrollo de la comunidad, esta no solo se limite a los impactos de las actividades sino también al apoyo y creación de una relación con dichas comunidades. Los asuntos clave del desarrollo a la comunidad incluyen creación de empleo mediante expansión y diversificación de las actividades económicas y el desarrollo tecnológico, inversiones sociales en la generación de riqueza mediante iniciativas de desarrollo económico local, programas de educación, promoción de la cultura y mejora de los servicios de salud.

Términos clave:

a. Participación activa de la comunidad (Consulta sobre priorización de inversiones a las comunidades, desarrollo inclusivo de comunidades, participación de asociaciones locales, alentar al voluntariado, contribuir a la formación de políticas de desarrollo local)

b. Educación y cultura (promover la educación en todos los niveles, promover la educación a grupos vulnerables, alentar a la formalización de la educación, promover actividades culturales, ayudar a conservar el patrimonio cultural, promover el uso de conocimientos tradicionales cuando sea pertinente)

c. Creación de empleo y desarrollo de habilidades (analizar el impacto de actividades sobre la creación de empleo, considerar el impacto a largo plazo sobre el empleo de las elecciones tecnológicas, creación de empleos directos, disminución del subempleo, promoción de programas de desarrollo de habilidades, prestar atención especial a grupos vulnerables en temas de empleo, creación de condiciones necesarias para la creación de empleo)

d. Desarrollo y acceso a la tecnología (contribuir al desarrollo de nuevas tecnologías, contribuir al desarrollo de tecnologías de bajo costo, cuando sea pertinente contribuir al desarrollo de tecnologías de escala local, alianzas con universidades o laboratorios, contribuir a la transferencia y difusión de tecnología)

e. Generación de riqueza e ingresos (considerar impactos económicos en las comunidades, iniciativas para impulsar la diversificación económica, dar preferencia a productos y servicios locales, ayudar a organizaciones a que operen dentro del marco jurídico adecuado, inclusión de organizaciones de bajos niveles de desarrollo, programas de alianzas estratégicas, fomentar uso eficiente de recursos, apoyo a organizaciones que promueven el empleo, ayuda a emprendedores, cumplir con responsabilidades tributarias, contribuir con la jubilación de las personas)

f. Salud (eliminar fuente de impacto negativas para la salud, promover acceso a medicinas, promoción de vida saludable, apoyo al acceso universal a servicios esenciales de salud y maternidad)

g. Inversión Social (proyectos de inversión social o en comunidades, evitar dependencia externa, evaluar iniciativas propias, alianzas estratégicas 
con gobiernos u ONGs en temas de inversión, promoción de programas que promuevan la alimentación de grupos vulnerables). 